\title{
CORRESPONDENCE
}

\section{Vasopressor therapy: not like antibiotics!}

\author{
Jan Bakker ${ }^{1,2,3^{*}}$ and Jukka Takala ${ }^{4}$
}

(c) 2016 Springer-Verlag Berlin Heidelberg and ESICM

\section{Dear Editor,}

We read with interest the publication by Lamontagne et al. [1]. The authors compared the effects of two treatment regimens with different mean arterial blood pressure (MAP) targets on various outcome parameters. In addition to the excellent accompanying editorial, we would like to emphasize two issues. First, the participating medical and nursing staff did not follow the objectives of the study and in general maintained a higher blood pressure than the actual target in both the low and high MAP groups. This required significantly higher dosing of vasopressors in the high-MAP group, resulting in adverse effects. This reflects an obvious common bias in the treatment of critically ill patients. Generally, physiologic variables in survivors of critical illness are closer to normal than those in non-survivors [2], leading to an erroneous thinking that maintaining more normal values creates survivors. This concept has been proven false in many studies and is theoretically flawed [3].

Second, we were surprised that in the discussion the use of vasopressors was repeatedly referred to as 'treatment' suggesting that vasodilatory shock automatically benefits from vasopressor treatment. For example, the authors defend the higher than target MAP in both groups: "...which suggests that nurses and physicians take great care to avoid under dosing vasopressors..." This suggests that the use of vasopressors should have target doses, analogous to dosing antibiotics to reach target plasma concentrations. However, in patients with MAP considered too low to maintain vital organ perfusion by the treatment team, the aim of therapy is not to administer vasopressors but to improve MAP to adequate levels. Various other interventions can be used or may be necessary to reach this goal varying from fluid resuscitation (or even fluid removal) to the use of various types of circulatory assist in the most extreme cases. Although this may seem semantic, we think this is conceptually relevant. Rather than using a specific MAP to limit the use of vasopressors, vasopressors should be targeted to maintain tissue perfusion. Fixed MAP targets in large groups of septic patients, who are inevitably heterogeneous, ignore the individual variability in (patho) physiology of patients and the needs to maintain tissue perfusion. The therapeutic interventions needed to maintain tissue perfusion are likely to change during the course of the disease. In this context, using fixed MAP targets appears simplistic. Rather, the targets should be individually adjusted.

The need for high dosages of vasopressors reflects severity of illness [4] and should urge the treatment team to reconsider the target: the level necessary to achieve and maintain sufficient tissue perfusion could even be much lower [5] than the ranges achieved in the study by Lamontagne et al. [1] and could help to avoid the adverse effects of high-dose vasopressors.

\section{Author details \\ ${ }^{1}$ Department of Intensive Care Adults, Erasmus MC University Medical Center, Rotterdam, The Netherlands. ${ }^{2}$ Division of Pulmonary, Allergy, and Critical Care Medicine, Columbia University Medical Center, New York, NY, USA. ${ }^{3}$ Depar- tamento de Medicina Intensiva, Facultad de Medicina, Pontificia Universidad Católica de Chile, Santiago, Chile. ${ }^{4}$ Department of Intensive Care Medicine, University Hospital Bern, Bern, Switzerland.}

Compliance with ethical standards

Conflicts of interest

The authors have no conflict of interest to declare.

Accepted: 15 March 2016

Published online: 25 April 2016 


\section{References}

1. Lamontagne F, Meade MO, Hebert PC, Asfar P, Lauzier F, Seely AJ, Day AG, Mehta S, Muscedere J, Bagshaw SM, Ferguson ND, Cook DJ, Kanji S, Turgeon AF, Herridge MS, Subramanian S, Lacroix J, Adhikari NK, Scales DC, Fox-Robichaud A, Skrobik Y, Whitlock RP, Green RS, Koo KK, Tanguay T, Magder S, Heyland DK, Canadian Critical Care Trials Group (2016) Higher versus lower blood pressure targets for vasopressor therapy in shock: a multicentre pilot randomized controlled trial. Intensive Care Med 42:542-550. doi:10.1007/s00134-016-4237-3

2. Bernardin G, Pradier C, Tiger F, Deloffre P, Mattei M (1996) Blood pressure and arterial lactate level are early indicators of short-term survival in human septic shock. Intensive Care Med 22:17-25
3. Kavanagh BP, Meyer LJ (2005) Normalizing physiological variables in acute illness: five reasons for caution. Intensive Care Med 31:1161-1167

4. Abid O, Akca S, Haji M, Vincent JL (2000) Strong vasopressor support may be futile in the intensive care unit patient with multiple organ failure. Crit Care Med 28:947-949

5. Dunser MW, Takala J, Brunauer A, Bakker J (2013) Re-thinking resuscitation: leaving blood pressure cosmetics behind and moving forward to permissive hypotension and a tissue perfusion-based approach. Crit Care 17:326 